\title{
The Disruptive bEhavior manageMEnt ANd prevention in hospitalized patients using a behaviORal intervention team (DEMEANOR) study protocol: a pragmatic, cluster, crossover trial
}

\section{Jay Morrison}

Vanderbilt University Medical Center

Michele Hasselblad

Vanderbilt University Medical Center

Ruth Kleinpell ( $\sim$ ruth.kleinpell@vanderbilt.edu )

Vanderbilt University School of Nursing https://orcid.org/0000-0003-0142-7527

Reagan Buie

Vanderbilt University Medical Center

Deborah Ariosto

Vanderbilt University Medical Center

Erin Hardiman

Vanderbilt University Medical Center

Stephen W Osborn

Vanderbilt University Medical Center

Christopher J Lindsell

Vanderbilt University Medical Center

Study protocol

Keywords: behavioral intervention team, disruptive behavior, hospitalized patients, advanced practice registered nurse, social work intervention

Posted Date: November 2nd, 2019

DOI: https://doi.org/10.21203/rs.2.16569/v1

License: (9) (1) This work is licensed under a Creative Commons Attribution 4.0 International License. Read Full License 
Version of Record: A version of this preprint was published at Trials on May 24th, 2020. See the published version at https://doi.org/10.1186/s13063-020-04278-2. 


\section{Abstract}

Background Disruptive behavior in hospitalized patients has become a priority area of safety concern for clinical staff, as well as having consequences for patient management and hospital course. Proactive screening and intervention of patients with behavioral co-morbidities has been reported to reduce disruptive behavior in some settings, but has not been studied in a rigorous way.

Methods The $\mathrm{D}$ isruptive $\mathrm{b} E$ havior manage ME nt AN d prevention in hospitalized patients using a behavi OR al intervention team (DEMEANOR) study is a pragmatic, cluster, cross-over trial being conducted. Each month, the behavioral intervention team, comprised of a psychiatric-mental health advanced practice nurse and a clinical social worker, with psychiatrist consultation as needed, rotates between an adult medicine unit and a mixed cardiac unit at Vanderbilt University Medical Center in Nashville, TN. The team proactively screens patients upon admission utilizing a protocol which includes a comprehensive chart review, and if indicated, a brief interview, seeking to identify those patients who possess risk factors indicative of either a potential psychological barrier to their own clinical progress or a potential risk for exhibiting disruptive, aggressive or self-injurious behavior during their hospitalization. Once identified, the team provides interventions aimed at mitigating these risks, educates and supports the patient care teams (nurses, physicians and others), and assists non-psychiatric staff in the management of patients who require behavioral health care. Patients who are both admitted to and discharged from either unit are included in the study. Anticipated enrollment is approximately 1790 patients. The two primary outcomes are i) a composite of objective measures related to the patients' disruptive, threatening or acting out behaviors, and ii) staff self-reported comfort with and confidence in their ability to manage patients exhibiting disruptive, threatening or acting out behavior. Secondary outcomes include patient length of stay, patient attendant (sitter) use, and the unit nursing staff retention.

Discussion This ongoing trial will provide evidence on the real-world effectiveness of a proactive behavioral intervention to prevent disruptive, threatening or acting out events in adult hospitalized patients.

\section{Background}

Patients who are hospitalized on nonbehavioral health units who exhibit concurrent psychiatric comorbidities can be at risk for increased length of hospital stay due to the complexities associated with treatment, as well as behaviors that can be difficult to address by staff. Research has substantiated that $20 \%$ to $40 \%$ of patients hospitalized on general medical-surgical units have a psychiatric diagnosis (Lee, 2017; Desan et al 2011). At our institution, $54 \%$ of all discharges on two medical surgical units during fiscal year 2017 had a behavioral health diagnosis. These patients had a $25 \%$ longer mean length of stay [6.6 days vs 5.31 without a behavioral health diagnosis], and $17 \%$ higher mean variable costs per discharge $(\$ 11,307$ vs $\$ 9,642)$, based on 104,843 total patient days. 
At the same time, an internal "Behavioral Health Care Knowledge and Skills" assessment of 623 staff nurses identified that $72 \%$ reported patients' behavior impacted their ability to provide care, $58 \%$ reported experiencing situational anxiety in caring for these patients, $56 \%$ reported caring for behavioral health patients daily to weekly (32\% report monthly), $50 \%$ reported feeling somewhat to very uncomfortable caring for these patients, and $44 \%$ feared for their personal safety as a result of patient disruptive behavior. These data demonstrate a large impact of patient behavior on the care that can be provided to them, and on the satisfaction and morale of nursing staff.

Our institutional data suggest nursing turnover rate is higher for those units with a higher burden of patients with behavioral comorbidities, and exit interviews suggest a link between turnover and the challenges of managing patients exhibiting disruptive behavior. Beyond turnover, there is considerable transfer from these units as nurses seek to improve their job satisfaction. Given the impact on patient care as well as those providing care, identifying effective strategies for preventing and management disruptive patient behavior has become a priority.

Several academic medical centers have reported numerous benefits with the use of a behavioral intervention team, which provides proactive consultation and liaison psychiatric service. Observed outcomes included decreased length of stay and decreased use of constant companions (sitters) (Lee, 2017; Sledge et al 2016; Desan et al 2011; Sledge et al 2015; Pestka et al 2012; Levenson et al 1992). However, most reports are anecdotal, and there is a lack of comparative effectiveness research supporting the impact of proactive measures to reduce disruptive behavior in hospitalized patients.

The aim of the present trial is to evaluate the impact of a behavioral intervention team on one medical surgical unit and a mixed cardiac care unit with a high proportion of patients with behavioral health comorbidities. We hypothesize that when compared to usual unit staffing, the addition of a dedicated, trained behavioral intervention team provides meaningful improvement in the prevention and management of disruptive behavior in the healthcare setting, and improvement in staff perceptions of their ability to manage patients exhibiting disruptive, threatening or acting out behavior.

\section{Methods}

This manuscript was written in accordance with Standard Protocol Items: Recommendations for Interventional Trials (SPIRIT) guidelines (Chan et al 2013).

\section{Design}

The DEMEANOR study is a single-center, pragmatic, cluster, crossover trial being beginning 1 March 2019 on one medical surgical unit and one mixed cardiac care clinical unit at Vanderbilt University Medical Center in Nashville, TN, USA. The behavioral intervention team is deployed to each unit in alternating months, as shown in Figure 1. Patients presenting to the unit during a month when the team is present 
contribute to the intervention arm, while those presenting to a unit when the team is not present contribute to the control arm.

\section{Study sites and period}

The two study units are a 27-bed mixed cardiac medical surgical unit and a 22-bed cardiac stepdown unit.

\section{Population}

All adult (aged $\geq 18$ years) patients admitted to either of the two units during the study period are eligible for the study. To prevent contamination between study arms, patients must both be admitted to and discharged from the unit during the study month; patients present on a unit at the crossover will not be included in the analysis. In addition, all nursing staff working in these units during the study are included.

\section{Enrollment/Randomization}

Patients presenting to and discharged from participating units are enrolled automatically. As a cluster, crossover trial, randomization was limited to which unit the intervention would be deployed to first. Unit nursing staff were invited to participate in online surveys by e-mail, with reminders through staff meetings; their participation is voluntary.

\section{Intervention}

The integration of the behavioral intervention team into the unit is not itself a research intervention. Institutional leadership planned to deploy one team as a demonstration project, and to scale it if successful. This provided an opportunity for rigorous evaluation of the team's effectiveness. The behavioral intervention team, a multidisciplinary team comprised of a psychiatric-mental health advanced practice nurse and a clinical social worker, with psychiatrist consultation as needed, is a modified version of an established model at Yale New Haven Hospital (Sledge et al 2016). The team proactively screens patients upon admission to the unit utilizing a protocol which includes a comprehensive chart review, and if indicated, a brief interview, seeking to identify those patients who possess risk factors indicative of either a potential psychological barrier to their own clinical progress or a potential risk for exhibiting disruptive, aggressive or self-injurious behavior during their hospitalization. Once identified, the team provides interventions aimed at mitigating these risks, through a variety of patient-specific interventions including: 
1. Psychiatric consultation and recommendations for symptom management

2. Behavioral plans of care for nurse/patient interaction

3. Psychosocial support and brief psychotherapeutic intervention

4. Curbside consultation for any member of the patient's healthcare team

5. Patient advocacy and care coordination

6. Psychiatric-specific disposition support, including both inpatient and outpatient psychiatric services

The team also provides education and support to the patient care teams (nurses, physicians and others), and assists non-psychiatric staff in the management of patients who require behavioral health care.

All patients on a unit when the behavioral intervention team is present will be eligible for behavioral intervention team services, including those who are already admitted when the team crosses into the unit. During a month when the behavioral intervention team is not present on a unit, the care and management of patients is not supported with proactive screening and management. Unit staff have access to all psychiatric or behavioral care routinely available in usual care. To ensure continuity of care, patients who continue to require psychiatric support at the time the behavioral intervention team rotates off the unit will continue to receive care equivalent to the usual care condition.

All unit staff will be exposed to the intervention throughout the study, although after the first month of the study only those staff on the unit initially randomized to the intervention will have been exposed. This provides an opportunity for a comparison between exposed and unexposed staff.

\section{Data collection}

Data are being collected for patients and for unit staff. Patient information is documented as a component of usual clinical care, and those needed for this study will be extracted from the electronic health record (EHR). Data include demographics, documentation of patient disruptive behavior and associated medication administration, and patient length of stay.

Anonymous surveys will be used to measure staff perceptions of their ability to manage disruptive patient behavior, and their experience with the behavioral intervention team. The behavioral health survey previously used at the organization is a 10-item instrument that assesses staff perceptions of workplace violence and safety (Table 1). For this study, we have adapted these instruments to be administered to nursing staff on the two participating units at three different time points: prior to the study, following the first one-month intervention period, and after the study period has been completed (Figure 1). The surveys 
will be conducted electronically using Research Electronic Data Capture (REDCap) (Harris et al 2009). REDCap allows for surveys completed by the same individuals to be linked while maintaining the anonymity of respondents by preventing access to the linkage tables held securely within the REDCap application.

\section{Primary outcomes}

This study has two primary outcomes: i) any documented interventions to manage disruptive, threatening or acting out behavior, and ii) staff self-reported comfort with and ability to manage patients exhibiting disruptive, threatening or acting out behavior. Documented evidence of intervention is defined as:

- violence control

- injurious behavior

- administration, including as needed administration of the following medications for behavior management: Quetiapine (Seroquel), Alprazolam (Xanax), Clonazepam (Klonopin, Haloperidol (Haldol), Lorazepam (Ativan), Olanzapine (Zyprexa), Risperidone (Risperdal), and Ziprasidone (Geodon).

\section{Secondary outcomes}

Secondary outcomes include the occurrence of each intervention separately, sitter use, use of physical or chemical restraints, patient length of stay, and unit nursing staff turnover (Table 2).

\section{Power calculation}

Approximately $17.5 \%$ of patients admitted to the two participating units required one or more of the behavioral interventions in the year prior to the study. To determine the number of months of data collection necessary to detect a reduction of $5 \%$ in the use of behavioral interventions between the intervention and control arm, we used the method described by Arnup et al. (2017). Based on patient length of stay and admit rates, we estimated between 89 and 105 patients would be eligible for inclusion each month (patients present on a unit at crossover will not be included in the analysis). Therefore, a cluster size of 90 was used. If the within period correlation is 0.1 and the within cluster within period correlation is also about 0.1 , we would achieve $80 \%$ power with 10 months of data collection (total sample size of 1790 patients exposed to either the control or intervention arm). Since these estimates are based on assumptions and it is possible that either a shorter or longer duration is needed, we have specified an interim analysis at 6 months to confirm the overall event rate, and to estimate the observed correlations. No comparisons between groups will occur at this time; accrued data will be used only for sample size re-estimation. 


\section{Data and safety monitoring and interim analysis}

The study involves the collection of data to compare patients who are or who are not exposed to a clinical care practice: the behavioral intervention team. As such, there is no research intervention. The PI and Co-PIs, in conjunction with the clinical nursing unit leadership, clinical nurse leaders and clinical nurse educators, will monitor for any potential impact of the study that may cause untoward impact on patients or staff on an ongoing basis throughout the duration of the study.

At six months, the data will be reviewed to determine how many additional months of data collection will be required to have sufficient power to detect the $5 \%$ reduction in behavioral outcomes that is being designated as minimally important. Based on power calculations, six months is the minimum period of time for which the study can run to find this difference. At six months, there will be sufficient information to more precisely estimate the magnitude of the within cluster and within cluster within period correlation to update the sample size estimate. Formal stopping rules for safety, efficacy or futility have not been designated.

Statistical analysis principles There are two main analyses. The first will compare outcomes between patients exposed to the behavioral intervention team and patients not exposed to the behavioral intervention team. The outcomes are quantitative variables that are either binary or ordinal in nature. Comparisons will use a logistic regression or proportional odds models, adjusted for covariates. The models will include cluster (unit) as a random effect, and will also include period.

The second analysis will compare staff perceptions between those exposed to the behavioral intervention and those not exposed to the behavioral intervention during the first month, and will also compare perceptions between before exposure to the behavioral intervention and after the study concludes. We will compare categorical variables using chi-square tests, and we will compare continuous variables using paired or independent samples t-tests as appropriate. For continuous data that are non-normally distributed, and for ordinal variables, we will use the Mann-Whitney U-test or the Wilcoxon test as appropriate. We may model the outcomes using appropriate regression techniques to explore factors associated with changes in staff perceptions.

\section{Presentation of the results}

After completion of enrollment and data analysis, the results of the trial will be communicated to the public through manuscript publication and submission of the results for the ClinicalTrials.gov database. Submission for publication will include public access to the full study protocol and statistical code. Authorship will be based on the International Committee of Medical Journal Editors guidelines (2018), and professional writers will not be used.

The flow of patients through the study will be presented in a flow diagram (Figure 1). Baseline characteristics will be presented by treatment group, as shown in Table 2). The percent of patient 
injurious behaviors reported will be determined by extracting reports of biting, kicking, throwing, etc. from the EHR. The percent of violence control measures used will be determined by extracting medications, restraints and sitters ordered from the EHR.

A composite of all documented indications of disruptive patient behavior including violence control nursing interventions, as needed (PRN) medication administration for behavior management, nursing problem of violence risk, or any injurious behavior will be extracted from the EHR. Each component of the primary outcome will also be reported separately, along with documented physical restraints for disruptive patient behavior.

The percent change in nurse comfort and confidence in their ability to manage patients exhibiting disruptive, threatening or acting out behavior, perceptions of workplace violence/safety, as well as perceived confidence, comfort and most helpful training/support received will be determined by comparing pre-, one month and post-implementation surveys.

The rate of unit nursing staff retention will be determined by extracting rates from internal human resources records. Patient length of stay will be determined by extracting data from the EHR.

\section{Discussion}

Upon completion, the DEMEANOR study will provide the most comprehensive data to date on the impact of a proactive behavioral intervention on disruptive, threatening or acting out events in adult hospitalized patients.

Several potential threats to the validity of the trial exist. As the primary and secondary variables are being collected from documentation in the EHR, the potential for incomplete data exists. Additional limitations in using EHR data in clinical research include ensuring data security and privacy, and overcoming challenges associated with linking clinical data from diverse systems (Cowie et al 2017). The use of sitters or virtual sitters and restraints are dependent on a variety of patient care conditions, and verifying those that pertain to disruptive patient behavior will be dependent on clinical documentation or specific ordering directives.

There is a possibility that patients hospitalized during the study period who did not receive a specific consult from the behavioral intervention team nonetheless benefited from the presence of the team. The study design does not allow a conclusive differentiation between a spillover effect on the units where the behavioral intervention team is implemented and a possible secular trend from other unmeasured influences on the primary or secondary outcomes. Consults on the control unit during the study period will be monitored to assess for the potential for an increase due to staff awareness and exposure to the behavioral intervention team during the crossover periods. Potential fluctuations in patient census during the trial, and the number of patients presenting with behavioral co-morbidities or those exhibiting disruptive behavior may also impact the study results. 
Nursing response rates to the pre-, one month and post-implementation surveys may impact the ability to evaluate change in perceptions of their ability to manage patients exhibiting disruptive, threatening or acting out behavior. Also, nursing retention rates are dependent on a number of factors including career opportunities which may not be related to nursing job dissatisfaction or to encountering patients with disruptive behavior.

The DEMEANOR study is a pragmatic trial, which provides the opportunity to evaluate the effectiveness of an intervention in real-life routine practice conditions (Patsopoulos 2011). Inherent in this design however is the potential for a lack of external validity. Additionally, with a single-center trial, there is limited ability to generalize the study results.

\section{Trial Status}

The DEMEANOR study is an ongoing, single-center, pragmatic, crossover trial that will provide comprehensive information on the impact of a proactive behavioral intervention on disruptive, threatening or acting out events in adult patients. The protocol is the current version (number one). Patient enrollment began on 1 March 2019 and enrollment is scheduled for completion on 30 December 2019. The study results will be submitted for publication in a peer-reviewed journal, and abstracts submitted for presentation at local, regional, and national professional conferences.

\section{Declarations}

Ethics approval and consent to participate: The trial was determined by the Institutional Review Board to pose minimal risk to patient participants because the proactive screening is being conducted as part of clinical care and is intended to enhance patient care. Furthermore, all patient data collected for the purposes of the study are generated as part of routine clinical care and are obtained by data extraction from the electronic health record. A waiver of informed consent for patients was requested because it was expected that substantial bias would be introduced by requiring consent and this would make it impracticable to conduct this research in a meaningful way; the Institutional Review Board approved the waiver of informed consent. Informed consent was required for clinical staff completing online surveys assessing their comfort with and confidence in their ability to manage patients exhibiting disruptive, threatening, or acting out behavior.

Consent for publication: not applicable

Availability of data and materials: On completion of the study, investigators external to the research team may request to collaborate on secondary analyses. With appropriate institutional review board approval and data use agreements in place, de-identified datasets may be released. All statistical code will be made publicly available with the analysis. The investigators plan to publish trial results without assistance from outside professional writers. The investigators have no publication restrictions.

Competing interests: The authors report no conflicts or competing interests

Page $10 / 18$ 
Funding:The project described was supported by the Vanderbilt Institute for Clinical and Translational Research (VICTR) Learning Healthcare System Platform under CTSA award No. UL1 TR002243 from the National Center for Advancing Translational Sciences. Its contents are solely the responsibility of the authors and do not necessarily represent official views of the National Center for Advancing Translational Sciences or the National Institutes of Health.

Authors' contributions: Each author has made substantial contributions to the conception or design of the work, implementation and analysis. Each author has approved the submitted version and has agreed to be personally accountable for their contributions to the study and to ensure that questions related to the accuracy or integrity of any part of the work are appropriately investigated, resolved, and the resolution documented in the literature. JM, MH, RK, RB and CL were involved in the conception and design of the work, JM, MH, RK, RB, EH, SWO, DA and CL were involved in implementation; DA, RB, and CL were involved in data review and oversight of the analysis. RK led the manuscript writing and revision work.

Acknowledgements: The trial is being conducted within the Vanderbilt Learning Healthcare System. The authors thank the patients, nurses, nurse practitioners and attending physicians at Vanderbilt University Medical Center for making this study possible.

Authors' information:

DEMEANOR Investigators:

Vanderbilt University Medical Center, Nashville, Tennessee, USA:

Jay Morrison, Michele Hasselblad, Ruth Kleinpell, Reagan Buie, Deborah Ariosto, Erin Hardiman, Stephen W. Osborn, Christopher Lindsell, Gordon Bernard, Todd Rice, Robin Steaban, Robert Dittus, Cheryl Gatto, Mary Lynn Dear, for the Vanderbilt Learning Healthcare System Investigators, Vanderbilt University

\section{References}

Arnup SJ, McKenzie JE, Hemming K, et al. Understanding the cluster randomized crossover design: a graphical illustration of the components of variation and a sample size tutorial. Trials 2017 18:381 DOI 10.1186/s13063-017-2113-2.

Chan AW, Tetzlaf JM, Altman DG, Laupacis A, et al. SPIRIT 2013 statement: defining standard protocol items for clinical trials. Annals of Internal Medicine 2013;158:200-7.

Cowie MR, Blomster JI, Curtis LH. Electronic health records to facilitate clinical research. Clin Res Cardiol 2017;106:1-9

Desan PH, Zimbrean PC, Weinstein AJ, et al. Proactive psychiatric consultation services reduce length of stay for admissions to an inpatient medical team. Psychosomatics. 2011;52:513-520. 
Harris PA, Taylor R, Thielke R, Payne J, Gonzalez N, Conde JG. Research electronic data capture (REDCap) -a metadata-driven methodology and workflow process for providing translational research informatics support. Journal of Biomedical Informatics, 2009;42:377-381.

International Committee of Medical Journal Editors Guidelines. http://www.icmje.org/icmjerecommendations.pdf (accessed June 30 2019)

Lee H. Yale behavioral intervention team (BIT) model study: results from the two-year implementation of a proactive CL psychiatric service at the Yale New Haven Hospital. Journal of Psychosomatic Research 2017; 97;157-158.

Levenson JL, Hamer RM, Rossiter LF. A randomized controlled study of psychiatric consultation guided by screening of general medical inpatients. American Journal of Psychiatry;1992;149:631-637.

Loucks J, Rutledge DN, Hatch B, Morrison V. Rapid response team for behavioral emergencies. Journal of the American Psychiatric Nurses Association 2010;16:93-10

Patsopoulos NA. A pragmatic view on pragmatic trials. Dialogues Clin Neurosci. 2011;13:217-224

Pestka EL, Hatteberg DA, Larson LA, Zwytart AM, Cox DL, Borgen EE. Enhancing safety in behavioral emergency situations. MedSurg Nursing 2012;21:335-341.

Sledge WH, Gueorguieva R, Desan P. Bozzo JE, Dorset J, Lee HB. Multidisciplinary proactive psychiatric consultation service: Impact on length of stay for medical inpatients. Psychotherapy and Psychosomatics. 2015;84:208-216.

Sledge WH, Bozzo J, White-McCullum BA, Lee H. The cost-benefit from the perspective of the hospital of a proactive psychiatric consultation service on inpatient general medicine services. Health Economics \& Outcome Research: Open Access. 2016;2:122. Doi:10.4172/2471-268x/1000122

Zicko JM, Schroeder RA, Bhers WS, Taylor AM, Spence DL. Behavioral emergency response team implementation improves patient safety, staff safety and staff collaboration. Worldviews on EvidenceBased Nursing 2017;15:377-384

\section{Tables}

Table 1. Behavioral health survey to assess staff perceptions of workplace violence and safety

1. Have you encountered patients exhibiting disruptive, threatening or acting out behavior? 
Yes

No

2. If yes, did their behavior in any way impact your ability to provide care?

Yes

No

3. Please specify how care was impacted: (Select all that apply)

Patient was non-compliant with care

Took time away from care with other patients

Disruptive to unit and other patients

Patient was combative

Other: please describe

4. In your current position, which of the following did you experience while caring for a patient exhibiting disruptive, threatening, or acting out behavior? Select all that apply

Physical (hit, kick, slapped, shoved, spit at, thrown objects, grabbed, bitten, or attacked)

Verbal (threatening, bullying, harassing, name calling, blaming, insulting, yelling, cursing, intimidation)

Elopement

Central line manipulation related to substance abuse Suicidal ideation 


\section{Self-injury}

Other (please describe)

5. Did you experience any of the following because of caring for a patient exhibiting disruptive, threatening, or acting out behavior?

$\begin{array}{llll}\text { Never } & \text { Rarely } & \text { Sometimes } & \text { Often }\end{array}$

Fear of personal safety

Fear for patient safety

Situational anxiety

A disruptive family member rather than a patient

Decreased job satisfaction

6. How comfortable are you with strategies in caring for patients exhibiting disruptive, threatening, or acting out behavior?

Very comfortable

Somewhat comfortable

Neutral

Somewhat uncomfortable

Very uncomfortable

7. What reporting structure do you utilize when you have experienced an event with a patient exhibiting disruptive, threatening, or acting out behavior? Select all that apply 
Consult service

Confidential online reporting system

First report of injury

Employee Assistance Program

Involve the primary team

Involve the one up leader

Do not report

None of the above

8. What has been the most beneficial training/support you have received in your current position at Vanderbilt to prepare you to provide care for patient's exhibiting disruptive, threatening, or acting out behavior? (Please rank order)

Behavioral health \& safety learning management education module

De-escalation and trauma informed care education

Unit based education

Psychiatric consult service

9. What do you think would be helpful for ongoing bedside support in the care of patients exhibiting disruptive, threatening, or acting out behavior? (rank order)

Behavioral management techniques

Safety restraints

Therapeutic communication

General education 
10. On a scale of 0 to 10 where 0 is the lowest and 10 is the highest level, rate your level of confidence in caring for patients exhibiting disruptive, threatening, or acting out behavior on your unit.

References: Adapted from Zicko JM et al. Behavioral emergency response team implementation improves patient safety, staff safety and staff collaboration. Worldviews on Evidence-Based Nursing 2017;15:377-384 and Loucks J, et al. Rapid response team for behavioral emergencies. Journal of the American Psychiatric Nurses Association 2010;16:93-10).

Table 2: Outcome Measures and Definitions 


\section{Primary}

Violence control measures utilized, or patient injurious behaviors reported
Composite of violence control nursing intervention; PRN medication administration of Quetiapine, ALPRAZolam, ClonazePAM, Haloperidol, LORazepam, OLANZapine, RisperiDONE, or Ziprasidon for behavior management; or nursing problem of violence risk or injurious behavior

Staff perceptions of workplace violence/safety, as well as perceived confidence, comfort and most helpful training/support received
Extraction of nursing documentation and medication administration from the electronic health record

A Behavioral Health Survey administered via Research Electronic Data Capture (REDCap) prior to intervention, one month after intervention began and at the conclusion of the intervention

\section{confidence in their ability to manage patients exhibiting disruptive, threatening or acting out behavior} Secondary

\begin{tabular}{|c|c|c|}
\hline $\begin{array}{l}\text { Rate of unit nursing } \\
\text { staff retention }\end{array}$ & $\begin{array}{l}\text { Amount of turnover (departure from the } \\
\text { organization) experienced on each study } \\
\text { unit while the study was conducted }\end{array}$ & $\begin{array}{l}\text { Extracted from Human } \\
\text { Resource records }\end{array}$ \\
\hline $\begin{array}{l}\text { Patient length of } \\
\text { stay* }\end{array}$ & $\begin{array}{l}\text { Number of days a patient spends in the } \\
\text { hospital from the time of the order to } \\
\text { admit to the time of discharge }\end{array}$ & $\begin{array}{l}\text { Extracted from the Electronic } \\
\text { Health Record }\end{array}$ \\
\hline $\begin{array}{l}\text { Individual } \\
\text { components of the } \\
\text { primary outcome }\end{array}$ & & \\
\hline $\begin{array}{l}\text { Violence control } \\
\text { intervention } \\
\text { PRN } \\
\text { medications for } \\
\text { behavior } \\
\text { management } \\
\text { - Nursing } \\
\text { problem of } \\
\text { violence risk or } \\
\text { injurious } \\
\text { behavior }\end{array}$ & As above & As above \\
\hline Use of restraints & $\begin{array}{l}\text { Use of physical or chemical restraints to control } \\
\text { patient behavior }\end{array}$ & $\begin{array}{l}\text { Extracted from the Electronic } \\
\text { Health Record }\end{array}$ \\
\hline $\begin{array}{l}\text { Use of } \\
\text { sitters/patient } \\
\text { attendants }\end{array}$ & $\begin{array}{l}\text { Use of sitters/patient attendants for patient } \\
\text { observation }\end{array}$ & $\begin{array}{l}\text { Extracted from the Electronic } \\
\text { Health Record }\end{array}$ \\
\hline
\end{tabular}

*Resource length of Stay (RLOS) - Indicates hospital resource/bed utilization, irrespective of the change in patient status. The measure excludes the time that encounters spend as an "Emergency Department" type patient but begins the instant a patient is given an order to admit as an inpatient and ends at discharge. This metric thus includes time a patient is in an emergency department bed, in the post anesthesia care unit, or other location but has an inpatient status. The metric is viewed by average, median, and adjusted by the total Casemix Index. 


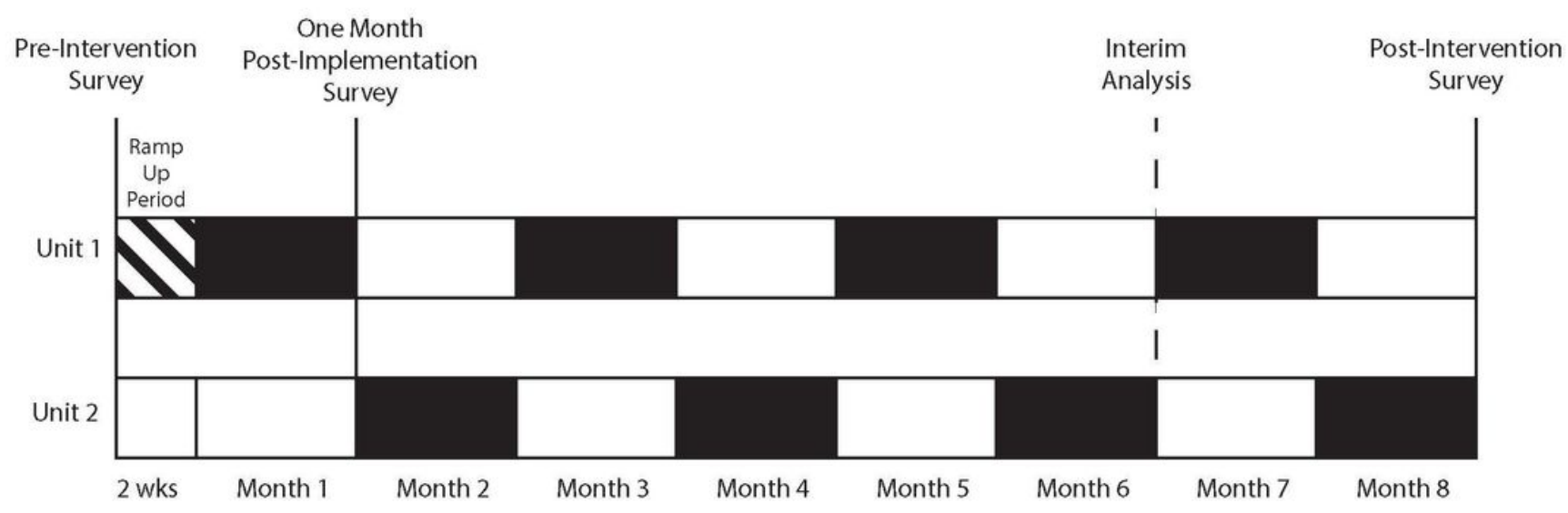

Figure 1

Depiction of the study implementation showing the ramp up period and the cross-over of the team between units. The timing of surveys and interim analysis is also shown. 